\title{
Bohr neighborhoods in generalized difference sets
}

\author{
John T. Griesmer \\ Department of Applied Mathematics and Statistics \\ Colorado School of Mines \\ Golden, Colorado, U.S.A. \\ jtgriesmer@gmail.com
}

Submitted: Aug 4, 2021; Accepted: Dec 15, 2021; Published: Feb 25, 2022

(C) The author. Released under the CC BY license (International 4.0).

\begin{abstract}
If $A$ is a set of integers having positive upper Banach density and $r, s, t$ are nonzero integers whose sum is zero, a theorem of Bergelson and Ruzsa says that the set $r A+s A+t A:=\left\{r a_{1}+s a_{2}+t a_{3}: a_{i} \in A\right\}$ contains a Bohr neighborhood of zero. We prove a natural generalization of this result for subsets of countable abelian groups and more summands.
\end{abstract}

Mathematics Subject Classifications: 11B13, 05B10, 37A45

\section{Bohr neighborhoods in iterated difference sets}

Let $\mathcal{S}^{1}:=\{z \in \mathbb{C}:|z|=1\}$ be the group of complex numbers with modulus 1 , with the usual topology and the operation of multiplication.

Let $G$ be a topological abelian group. If $A, B \subseteq G$, we write $A+B$ for the sumset $\{a+b: a \in A, b \in B\}$ and $A-A$ for the difference set $\left\{a-a^{\prime}: a, a^{\prime} \in A\right\}$. A character of $G$ is a continuous homomorphism $\chi: G \rightarrow \mathcal{S}^{1}$. Of course, when $G$ is discrete, every homomorphism to $\mathcal{S}^{1}$ is continuous. The set $\widehat{G}$ of characters of $G$ forms an abelian group under pointwise multiplication. When $G$ is discrete and $\widehat{G}$ is given the topology of pointwise convergence, $\widehat{G}$ is a compact abelian group. When $G$ is compact Hausdorff and $\widehat{G}$ is given the topology of uniform convergence, $\widehat{G}$ is discrete.

A trigonometric polynomial is linear combination of characters, i.e. a function of the form $\sum_{j=1}^{d} c_{j} \chi_{j}$, where $\chi_{j} \in \widehat{G}$ and $c_{j} \in \mathbb{C}$. A function $\psi$ on $G$ is (uniformly) almost periodic if it is a uniform limit of trigonometric polynomials.

Let $d \in \mathbb{N}$ and $\chi_{1}, \ldots, \chi_{d} \in \widehat{G}$. The basic Bohr neighborhood of 0 in $G$ having rank $d$ and radius $\varepsilon$ determined by $\left\{\chi_{1}, \ldots, \chi_{d}\right\}$ is

$$
\operatorname{Bohr}\left(\chi_{1}, \ldots, \chi_{d} ; \varepsilon\right):=\left\{g:\left|\chi_{i}(g)-1\right|<\varepsilon \text { for all } i=1, \ldots, d\right\} .
$$

A we say that $B$ is a Bohr neighborhood of 0 if it contains $\operatorname{Bohr}\left(\chi_{1}, \ldots, \chi_{d} ; \varepsilon\right)$ for some $d \in \mathbb{N}, \chi_{i} \in \widehat{G}$, and $\varepsilon>0$. 
Remark 1.1. If $\psi$ is a real valued trigonometric polynomial with $\psi(0)>0$, it is easy to verify that $\operatorname{supp}(\psi):=\{g \in G: \psi(g) \neq 0\}$ is a Bohr neighborhood of 0 . The same holds when $\psi$ is almost periodic, since every such $\psi$ is a uniform limit of trigonometric polynomials.

A classical theorem of Bogoliouboff [11] states that if $G$ is a countable abelian group and $G=C_{1} \cup C_{2} \cup \cdots \cup C_{r}$ is a finite cover of $G$, then for at least one $i$, the iterated difference set $\left(C_{i}-C_{i}\right)-\left(C_{i}-C_{i}\right):=\left\{(x-y)-(z-w): x, y, z, w \in C_{i}\right\}$ contains a basic Bohr neighborhood of 0 with rank and radius depending only on $r$. Note that Bogoliouboff's theorem implies that if finitely many translates of $C$ cover $G$, then $(C-C)-(C-C)$ contains a Bohr neighborhood of 0 . Følner [13] generalizes this to say that $(A-A)-(A-A)$ contains a Bohr neighborhood of 0 under the weaker hypothesis that $d^{*}(A)>0$, where $d^{*}$ denotes upper Banach density (see $\S 2$ for the definition). See $\S 5$ in Part II of [17] for exposition of Bogoliouboff's and Følner's results.

Bergelson and Ruzsa extended Følner's theorem as follows.

Theorem 1.2 ([8], Theorem 6.1). Let $r, s, t$ be nonzero integers with $r+s+t=0$, and let $A \subseteq \mathbb{Z}$ have $d^{*}(A)>0$. Then $r A+s A+t A:=\left\{r a_{1}+s a_{2}+t a_{3}: a_{i} \in A\right\}$ contains $a$ basic Bohr neighborhood of 0 , with rank and radius depending only on $r, s, t$, and $d^{*}(A)$.

Our main result is Theorem 1.3, a partial generalization of Theorem 1.2 to arbitrary countable abelian groups and more summands. We say "partial generalization" because our Theorem 1.3 does not bound the rank and radius of the Bohr neighborhood.

Let $G$ be a countable (discrete) abelian group. Let $q$ be the exponent of $G$, meaning the least $m \in \mathbb{N}$ such that $m g=0$ for every $g \in G$. If there is no such $m$, we say $G$ has exponent 0. Often "exponent 0" and "infinite exponent" are used interchangeably; in this article we say that $\mathbb{Z}, \mathbb{Z}^{2}$, etc. have exponent 0 .

If $\vec{c}=\left(c_{1}, \ldots, c_{d}\right) \in \mathbb{Z}^{d}$ and $A \subseteq G$, we define

$$
\vec{c} \cdot A:=\left\{\sum_{i=1}^{d} c_{i} a_{i}: a_{1}, \ldots, a_{d} \in A \text { are mutually distinct }\right\} \cup\{0\} .
$$

Theorem 1.3. Let $d \in \mathbb{N}, d \geqslant 3$, and let $\vec{c}=\left(c_{1}, \ldots, c_{d}\right) \in \mathbb{Z}^{d}$ be such that

(1) $c_{i} G:=\left\{c_{i} g: g \in G\right\}$ has finite index in $G$ for each $i$,

(2) $\left(c_{d-1}+c_{d}\right) G$, has finite index in $G$, and

(3) $q$ divides $\sum c_{i}$ (meaning $\sum c_{i}=0$ when $G$ has exponent 0 ).

If $A \subseteq G$ has $d^{*}(A)>0$, then $\vec{c} \cdot A$ is a Bohr neighborhood of 0 in $G$.

Note: hypothesis $(2)$ can be generalized to "there exists $i<j \leqslant d$ such that $\left(c_{i}+c_{j}\right) G$ has finite index in $G$," since the $c_{i}$ can be reordered without changing the value of $\vec{c} \cdot A$.

Letting $p$ be an odd prime, $G=\mathbb{F}_{p}^{\omega}:=$ the direct sum of countably many copies of $\mathbb{Z} / p \mathbb{Z}$, and $c_{1}=\cdots=c_{d}=1$, we get the following corollary as a special case of Theorem 1.3 . 
Corollary 1.4. If $p$ is an odd prime, $p$ divides $d \in \mathbb{N}$, and $A \subseteq \mathbb{F}_{p}^{\omega}$ has $d^{*}(A)>0$, then

$$
\left\{a_{1}+\cdots+a_{d}: a_{i} \in A \text { are mutually distinct }\right\} \cup\{0\}
$$

is a Bohr neighborhood of 0 .

Remark 1.5. While Theorem 1.2 and the theorems of Bogoliouboff and Følner do not specify that the sums $\left(a_{1}-a_{2}\right)-\left(a_{3}-a_{4}\right)$ be formed with mutually distinct $a_{i}$, their proofs easily yield the same conclusion under this additional specification. We impose distinctness of the $a_{i}$ here for the sake of an application of Theorem 1.3 in [20].

Remark 1.6. We are currently unable to decide whether hypothesis (2) can be omitted in Theorem 1.3. It is redundant when $d=3$, as hypothesis (3) implies $c_{2}+c_{3}=-c_{1}$ in that case, and hypothesis (1) implies $c_{1} G$ has finite index in $G$. See $\S 4$ for further discussion. The other hypotheses cannot be omitted, as the following examples demonstrate.

(i) The assumption $d \geqslant 3$ cannot be weakened to $d \geqslant 2$ : setting $d=2$ and $c_{1}=1$, $c_{2}=-1$, the set $\vec{c} \cdot A$ is simply $A-A$. An example of a set $A \subseteq \mathbb{Z}$ having $d^{*}(A)>0$ such that $A-A$ does not contain a Bohr neighborhood of 0 is provided by the main result of [24] - see [22] for an explanation of why [24] implies this. Such an example in $\mathbb{F}_{2}^{\omega}$ is constructed in [14]. In [19] it is shown that there is an $A \subseteq \mathbb{Z}$ with $d^{*}(A)>0$ where $A-A$ does not contain a Bohr neighborhood of any $n \in \mathbb{Z}$.

(ii) The hypothesis that $c_{i} G$ has finite index in $G$ cannot be omitted. To see this, we use the fact that for each prime $p$ and $\varepsilon>0$, the group $G=\mathbb{F}_{p}^{\omega}$ contains a set $A$ having $d^{*}(A)>\frac{1}{2}-\frac{1}{2 p}-\varepsilon$ such that $A-A$ does not contain a Bohr neighborhood of any element of $G$ (see [18]). Setting $c_{1}=p, c_{2}=1$ and $c_{3}=-1$, we have $c_{1}+c_{2}+c_{3}=p$, but $\vec{c} \cdot A \subseteq p A+A-A=A-A$.

(iii) The hypothesis that $q \mid \sum c_{i}$ cannot be omitted. For example, in $\mathbb{Z}$, the set $A$ of odd integers has $d^{*}(A)=1 / 2$, and $A-A+A=A$, which does not contain a Bohr neighborhood of 0 . Similarly in any cyclic group $\mathbb{Z} / N \mathbb{Z}$ of order $N, A:=\{1\}$ has $d^{*}(A)=\frac{1}{N}$ and $A-A+A=\{1\}$, which does not contain a Bohr neighborhood of 0 . Nevertheless, $A-A+A$ necessarily contains Bohr neighborhoods of many elements of $A$ whenever $d^{*}(A)>0$; see [22], [10].

The next lemma deals with a technicality arising from our approach. We first observe that if $\chi \in \widehat{G}$ and $a, b \in G$, then

$$
|\chi(a-b)-1| \leqslant|\chi(a)-1|+|\chi(b)-1| .
$$

This can be verified by writing

$$
\begin{aligned}
\chi(a-b)-1 & =\chi(a-b)-\chi(a)+\chi(a)-1 \\
& =\chi(a)(\overline{\chi(b)-1})+\chi(a)-1
\end{aligned}
$$

and applying the triangle inequality. 
Lemma 1.7. If $B$ is a Bohr neighborhood of 0 in an abelian group $G$ and $c \in \mathbb{Z}$ is such that $c G$ has finite index in $G$, then $c B$ is a Bohr neighborhood of 0 in $G$.

Proof. This is somewhat cumbersome to verify directly, but is an easy consequence of Bogoliouboff's theorem. Let $\varepsilon>0$ and let $B=\operatorname{Bohr}\left(\chi_{1}, \ldots, \chi_{d}, \varepsilon\right)$ be a basic Bohr neighborhood of 0 in $G$. Two applications of inequality (1.2) show that $B$ contains $(A-A)-(A-A)$, where $A:=\operatorname{Bohr}\left(\chi_{1}, \ldots, \chi_{d}, \varepsilon / 4\right)$. One can verify directly that finitely many translates of $A$ cover $G$, so finitely many translates of $c A$ cover $c G$. Since $c G$ has finite index in $G$, we get that finitely many translates of $c A$ cover $G$. Now $c B$ contains $(c A-c A)-(c A-c A)$, which must contain a Bohr neighborhood of 0 , by Bogoliouboff's theorem.

We adopt the usual notation for integrals: $\int f d \mu$ is the integral of $f$ with respect to $\mu$; we write $\int f(x) d \mu(x)$ if we need to specify a variable. When $\mu$ is Haar measure on a compact abelian group $K$, we may write $\int f(x) d x$ in place of $\int f(x) d \mu(x)$, to shorten the appearance of iterated integrals.

We will prove Theorem 1.3 by proving that there is an almost periodic function $\phi$ with $\phi(0)>0$ and $c_{d} \operatorname{supp}(\phi) \subseteq \vec{c} \cdot A$ (cf. Remark 1.1 and Lemma 1.7). The following lemma specifies the form of $\phi$.

Lemma 1.8. Let $A \subseteq G$ and let $c_{1}, \ldots, c_{d}$ satisfy the hypotheses of Theorem 1.3. Then there is a compact abelian group $K$ with Haar probability measure $m$, an m-measurable $\tilde{f}: K \rightarrow[0,1]$ with $\int \tilde{f} d m=d^{*}(A)$, and a homomorphism $\rho: G \rightarrow K$ with $\overline{\rho(G)}=K$ such that $\phi: G \rightarrow \mathbb{C}$ defined by

$\phi(t):=\int_{K^{d}} \tilde{f}\left(k+c_{d} s_{1}\right) \tilde{f}\left(k+c_{d} s_{2}\right) \cdots \tilde{f}\left(k+c_{d} s_{d-1}\right) \tilde{f}\left(k+\rho(t)-\sum_{i=1}^{d-1} c_{i} s_{i}\right) d k d s_{1} d s_{2} \ldots d s_{d-1}$

satisfies $c_{d} \operatorname{supp}(\phi) \subseteq \vec{c} \cdot A$.

Proof of Theorem 1.3. Assuming Lemma 1.8, it suffices to prove that the $\phi$ defined therein is almost periodic and satisfies $\phi(0)>0$ (cf. Remark 1.1).

To prove almost periodicity, let $\varepsilon>0$, and let $p: K \rightarrow[0,1]$ be a trigonometric polynomial with $\|\tilde{f}-p\|_{L^{2}(m)}<\varepsilon / d$. Such a $p$ exists as the trigonometric polynomials are uniformly dense in space of continuous functions on $K$, and the continuous functions are dense in $L^{2}(m)$. Set

$\phi_{\varepsilon}(t):=\int_{K^{d}} p\left(k+c_{d} s_{1}\right) p\left(k+c_{d} s_{2}\right) \cdots p\left(k+c_{d} s_{d-1}\right) p\left(k+\rho(t)-\sum_{i=1}^{d-1} c_{i} s_{i}\right) d k d s_{1} d s_{2} \ldots d s_{d-1}$.

This $\phi_{\varepsilon}$ is a trigonometric polynomial, as can be verified by evaluating the integral when each instance of $p$ is replaced by a character of $K$, and noting that $\phi_{\varepsilon}$ is a linear combination of such evaluations. Now we will show that $\left|\phi(t)-\phi_{\varepsilon}(t)\right|<\varepsilon$ for all $t$. We apply the identity

$$
\left(a_{1} a_{2} \cdots a_{d}\right)-\left(b_{1} b_{2} \cdots b_{d}\right)=\sum_{j=1}^{d} a_{1} \cdots a_{j-1}\left(a_{j}-b_{j}\right) b_{j+1} \cdots b_{d}
$$


with $a_{j}=\tilde{f}\left(k+c_{d} s_{j}\right), b_{j}=p\left(k+c_{d} s_{j}\right)$ for $j<d, a_{d}=\tilde{f}\left(k+\rho(t)-\sum_{i=1}^{d-1} c_{i} s_{i}\right), b_{d}=$ $p\left(k+\rho(t)-\sum_{i=1}^{d-1} c_{i} s_{i}\right)$. Thus

$$
\phi(t)-\phi_{\varepsilon}(t)=\sum_{j=1}^{d} \int_{K^{d}} a_{1} \cdots a_{j-1}\left(a_{j}-b_{j}\right) b_{j+1} \cdots b_{d} d k d s_{1} d s_{2} \ldots d s_{d-1} .
$$

The $a_{j}$ and $b_{j}$ are all uniformly bounded by 1 , so the inequality $\|\tilde{f}-p\|_{L^{2}(m)}<\varepsilon / d$ and Cauchy-Schwarz lets us bound the inner integral in (1.3) by $\varepsilon / d$. We therefore have $\left|\phi(t)-\phi_{\varepsilon}(t)\right|<\varepsilon$ for all $t$. Since $\varepsilon>0$ was arbitrary and $\phi_{\varepsilon}$ is a trigonometric polynomial, this proves that $\phi$ is almost periodic.

To prove that $\phi(0)>0$, note that the function on $K^{d-1}$ defined by

$$
\left(s_{1}, \ldots, s_{d-1}\right) \mapsto \int \tilde{f}\left(k+s_{1}\right) \cdots \tilde{f}\left(k+s_{d-1}\right) \tilde{f}\left(k-\sum_{i=1}^{d-1} c_{i} s_{i}\right) d k
$$

is continuous, and is equal to $\int \tilde{f}(k)^{d} d k$ when $s_{1}=s_{2}=\cdots=s_{d-1}=0$. The integrand in the definition of $\phi$ is therefore positive for a neighborhood of values $\left(s_{1}, \ldots, s_{d-1}\right)$ near 0 . Consequently, the integral defining $\phi$ does not vanish at $t=0$.

Remark 1.9. The argument in the preceding paragraph is a key ingredient in ergodic theoretic proofs of Roth's theorem on three-term arithmetic progressions. In fact, Roth's theorem is a corollary of our proofs, although we do not discuss this implication here. The proof of Theorem 6.1 in [8] (Theorem 1.2 above) uses Roth's theorem in a key step.

\section{Convolutions supported on sumsets}

In this section we state Theorem 2.3, an ergodic theoretic version of Lemma 1.8, and use it to prove the lemma. Theorem 2.3 will be proved in $\S 3.7$, after some preliminary machinery is developed.

Throughout this section we fix an arbitrary countable abelian group $G$.

Definition 2.1. A sequence $\mathbf{F}=\left(F_{n}\right)_{n \in \mathbb{N}}$ of finite subsets of $G$ is a Følner sequence if for all $g \in G, \lim _{n \rightarrow \infty} \frac{\left|F_{n} \Delta\left(g+F_{n}\right)\right|}{\left|F_{n}\right|}=0$. If $\mathbf{F}$ is a Følner sequence and $A \subseteq G$ the upper density of $A$ with respect to $\mathbf{F}$ is $\bar{d}_{\mathbf{F}}(A): \limsup _{n \rightarrow \infty} \frac{\left|A \cap F_{n}\right|}{\left|F_{n}\right|}$. The upper Banach density of $A$ is $d^{*}(A):=\sup \left\{d_{\mathbf{F}}(A): \mathbf{F}\right.$ is a $\mathbf{F} \varnothing l n e r$ sequence $\}$.

It is well known ${ }^{1}$ that every countable abelian group admits a Følner sequence.

Our first step in the proof of Lemma 1.8 is to find a kind of convolution supported $\vec{c} \cdot A$. For the sake of explanation, we first examine $A+B$ in place of $\vec{c} \cdot A$.

\footnotetext{
${ }^{1} \mathrm{~A}$ standard proof is to enumerate $G$ as $\left(g_{n}\right)_{n \in \mathbb{N}}$ and for each $n$, find a finite $F_{n}$ in the subgroup $\left\langle g_{1}, \ldots, g_{n}\right\rangle$ generated by $g_{1}, \ldots, g_{n}$, so that $\left|F_{n} \triangle\left(F_{n}+g_{i}\right)\right|<\frac{1}{n}\left|F_{n}\right|$ for all $i \leqslant n$. Such $F_{n}$ can be found by writing the finitely generated group $\left\langle g_{1}, \ldots g_{n}\right\rangle$ as $\mathbb{Z}^{d} \times K$, where $K$ is a finite group, and taking $F_{n}=\left[0, M_{n}\right]^{d} \times K$ for sufficiently large $M_{n}$.
} 
Given $A, B \subseteq G$, consider their characteristic functions $1_{A}$ and $1_{B}$. Note that a fixed $g$ lies in $A+B$ if and only if there is an $h \in G$ such that $1_{A}(h) 1_{B}(g-h)>0$. Often the existence of such an $h$ is proved by averaging, i.e. proving that there is a Følner sequence $\mathbf{F}$ with $\lim _{N \rightarrow \infty} \frac{1}{\left|F_{n}\right|} \sum_{h \in F_{n}} 1_{A}(h) 1_{B}(g-h)>0$. This limit is a kind of convolution of $1_{A}$ and $1_{B}$.

The next lemma shows exhibits a similar kind of convolution supported on $\vec{c} \cdot A$, written in a form suited to the hypotheses of Theorem 1.3.

Lemma 2.2. Let $\vec{c} \in \mathbb{Z}^{d}$ satisfy hypothesis (3) of Theorem 1.3, let $A \subseteq G$, and write $1_{A}$ for its characteristic function. Define $J: G^{d+1} \rightarrow[0,1]$ and $J_{0} \subseteq G$ by

$$
\begin{aligned}
& J\left(h, g_{1}, \ldots, g_{d-1} ; t\right):=1_{A}\left(h+c_{d} g_{1}\right) 1_{A}\left(h+c_{d} g_{2}\right) \cdots 1_{A}\left(h+c_{d} g_{d-1}\right) 1_{A}\left(h+t-\sum_{i=1}^{d-1} c_{i} g_{i}\right) \\
& J_{0}:=\left\{t \in G: \exists h, g_{1}, \ldots, g_{d-1} \in G \text { with } J\left(h, g_{1}, \ldots, g_{d-1} ; t\right)>0, c_{i} g_{i} \text { mutually distinct }\right\},
\end{aligned}
$$

we have

$$
c_{d} J_{0} \subseteq \vec{c} \cdot A
$$

Furthermore, setting

$$
\begin{aligned}
& \tilde{J}\left(g_{1}, \ldots, g_{d-1} ; t\right):=d^{*}\left(\left\{h: J\left(h, g_{1}, \ldots, g_{t-1} ; t\right)>0\right\}\right), \\
& \tilde{J}_{0}:=\left\{t \in G: \exists g_{1}, \ldots, g_{d-1} \in G \text { with } \tilde{J}\left(g_{1}, \ldots, g_{d-1} ; t\right)>0, c_{i} g_{i} \text { mutually distinct }\right\},
\end{aligned}
$$

we have $c_{d} \tilde{J}_{0} \subseteq \vec{c} \cdot A$.

Proof. We first prove the containment (2.1). Suppose $h, g_{i}$, and $t \in G$ are such that $J\left(h, g_{1}, \ldots, g_{d-1} ; t\right)>0$. Then each of $a_{i}:=h+c_{d} g_{i}$, and $a_{d}:=h+t-\sum_{i=1}^{d-1} c_{i} g_{i}$ lie in $A$, and

$$
c_{1} a_{1}+\cdots+c_{d} a_{d}=\left(c_{1}+\cdots+c_{d}\right) h+c_{d}\left(\sum_{i=1}^{d-1} c_{i} g_{i}\right)+c_{d}\left(t-\sum_{i=1}^{d-1} c_{i} g_{i}\right)=c_{d} t
$$

so $c_{d} t \in \vec{c} \cdot A$.

The second assertion of the lemma follows by observing that if $\tilde{J}\left(g_{1}, \ldots, g_{d-1} ; t\right)>0$, then there are infinitely many $h$ with $J\left(h, g_{1}, \ldots, g_{d-1} ; t\right)>0$.

Lemma 1.8 will be deduced from the following ergodic theoretic version; see $\S 3$ for definitions. These statements are connected by Proposition 2.5, a standard correspondence principle.

Theorem 2.3. Let $(X, \mu, T)$ be an ergodic measure preserving $G$-system and let $c_{1}, \ldots, c_{d}$ satisfy hypotheses (1)-(3) of Theorem 1.3. If $f_{1}, \ldots, f_{d}: X \rightarrow[0,1]$ are measurable functions, $L: G^{d} \rightarrow[0,1]$ is defined as

$$
L\left(g_{1}, \ldots, g_{d-1} ; t\right):=\int\left(T_{t-\sum_{j<d} c_{j} g_{j}} f_{d}\right) \prod_{j<d} T_{c_{d} g_{j}} f_{j} d \mu
$$


and $I(t)$ is defined as the iterated limit

$$
I(t):=\lim _{n_{1} \rightarrow \infty} \ldots \lim _{n_{d-1} \rightarrow \infty} \frac{1}{\left|F_{n_{1}}\right| \cdots\left|F_{n_{d-1}}\right|} \sum_{\substack{g_{1} \in F_{n_{1}} \\ g_{d-1} \ddot{\in F}_{n_{d-1}}}} L\left(g_{1}, \ldots, g_{d-1} ; t\right)
$$

then there are

- a compact metrizable abelian group $K$ with Haar probability measure $m$,

- a homomorphism $\rho: G \rightarrow K$ having $\overline{\rho(G)}=K$,

- functions $\tilde{f}_{i}: K \rightarrow[0,1]$ with $\int \tilde{f}_{i} d m=\int f_{i} d \mu$ for $i \leqslant d$

such that

$$
I(t)=\int \tilde{f}_{d}\left(k+\rho(t)-\sum_{j<d} c_{j} s_{j}\right) \prod_{j<d} \tilde{f}_{j}\left(k+c_{d} s_{j}\right) d k d s_{1} \ldots d s_{d-1} .
$$

Furthermore, the $\tilde{f}_{i}$ depend only on the $f_{i}$, so if $f_{1}=\cdots=f_{d}$, then $\tilde{f}_{1}=\cdots=\tilde{f}_{d}$.

We prove Theorem 2.3 in $\S 3.7$.

Remark 2.4. With $L$ and $I$ as in Theorem 2.3, let

$L_{0}:=\left\{t \in G: \exists g_{1}, \ldots, g_{d-1}\right.$ with $L\left(g_{1}, \ldots, g_{d-1} ; t\right)>0$ and $c_{i} g_{i}$ mutually distinct $\}$.

The containment $\{t \in G: I(t)>0\} \subseteq L_{0}$ follows easily from the definitions of $L$ and $I$.

The connection between Theorem 2.3 and Lemma 1.8 is made through the following variant of Furstenberg's correspondence principle. It is a special case of Theorem 2.8 of [5]; cf. the discussion following Proposition 2.3 of [3].

Proposition 2.5. Let $A \subseteq G$. There is an ergodic $G$-system $(X, \mu, T)$ and a measurable set $\tilde{A} \subseteq X$ with $\mu(\tilde{A})=d^{*}(A)$ such that for all $g_{1}, g_{2}, \ldots, g_{d} \in G$, we have

$$
d^{*}\left(\left\{h: 1_{A}\left(h+g_{1}\right) 1_{A}\left(h+g_{2}\right) \ldots 1_{A}\left(h+g_{d}\right)>0\right\}\right) \geqslant \int \prod_{i=1}^{d} 1_{\tilde{A}}\left(T_{g_{i}} x\right) d \mu(x)
$$

To deduce our Proposition 2.5 from Theorem 2.8 of [5], note that our $A$ is their $E$, our $\tilde{A}$ is their $A$, the expressions $g_{i}^{-1} E^{w_{i}}$ in equation (2.6) there can be written as $E^{w_{i}}-g_{i}$ when $G$ is abelian, and the left hand side of (2.6) here can be written as $d^{*}\left(\left(A-g_{1}\right) \cap \cdots \cap\left(A-g_{d}\right)\right)$.

Setting $g_{d}=t-\sum_{j<d} c_{j} g_{j}$ in Proposition 2.5 immediately yields the following corollary.

Corollary 2.6. If $A \subseteq G, c_{i} \in \mathbb{Z}$, and $\tilde{J}$ is defined as in Lemma 2.2, then there is an ergodic $G$-system $(X, \mu, T)$ and $f: X \rightarrow[0,1]$ such that

$$
\tilde{J}\left(g_{1}, \ldots, g_{d-1} ; t\right) \geqslant L\left(g_{1}, \ldots, g_{d-1} ; t\right) \quad \text { for all } g_{i}, t \in G,
$$

where $L$ is as defined in (2.2), with $f_{1}=\cdots=f_{d}=f$. 
Proof of Lemma 1.8. Let $G$ be a countable abelian group, let $c_{1}, \ldots, c_{d} \in \mathbb{Z}$ satisfy hypotheses (1)-(3) in Theorem 1.3, and let $A \subseteq G$ have $d^{*}(A)>0$. Let $\tilde{J}$ and $\tilde{J}_{0}$ be as in Lemma 2.2 , so that $c_{d} \tilde{J}_{0} \subseteq \vec{c} \cdot A$. Apply Corollary 2.6 to find an ergodic $G$-system $(X, \mu, T)$ and $f: X \rightarrow[0,1]$ with $\int f d \mu=d^{*}(A)$ satisfying inequality (2.7). This inequality implies $L_{0} \subseteq \tilde{J}_{0}$, where $L_{0}$ is defined in (2.5). By Theorem 2.3 and Remark 2.4, we have that $\{t \in G: I(t)>0\} \subseteq L_{0}$. Combining the preceding containments, we have

$$
c_{d}\{t \in G: I(t)>0\} \subseteq \vec{c} \cdot A .
$$

The form of $I(t)$ stated in the conclusion of Theorem 2.3 means we can take $\phi(t):=I(t)$ to satisfy the conclusion of Lemma 1.8 .

\section{$3 \quad$ Ergodic theoretic machinery}

\subsection{Measure preserving systems}

We use the standard ergodic theoretic setup for studying configurations in dense subsets of abelian groups; see any of [15], [12], [26], [23] for background. Our analysis here closely resembles that in $\S 2$ and $\S 3$ of $[1]$.

We again fix a countable abelian group $G$ throughout this section.

A triple $(X, \mu, T)$ is a measure preserving $G$-system (or "G-system") if $(X, \mu)$ is a standard probability measure space (cf. $\$ 1.4$ of [23]) and $G$ acts on $X$ by transformations $T_{g}$ which preserve $\mu$. If $f \in L^{2}(\mu)$ and $g \in G$, we write $T_{g} f$ for $f \circ T_{g}$. This defines an action of $G$ on $L^{2}(\mu)$ by unitary operators $T_{g}$.

We write $A \sim_{\mu} B$ if $\mu(A \triangle B)=0$, and $f \equiv_{\mu} g$ if $f, g: X \rightarrow \mathbb{C}$ agree $\mu$-almost everywhere. We say that

- $f$ is $T$-invariant if $f \equiv_{\mu} T_{g} f$ all $g \in G$;

- $B \subseteq X$ is $T$-invariant if $B \sim_{\mu} T_{g} B=0$ for every $g \in G$;

- $(X, \mu, T)$ is ergodic if the only $\mu$-measurable $T$-invariant sets $B$ have $\mu(B)=0$ or $\mu(B)=1$.

We will use the Mean Ergodic Theorem for unitary actions; see [23, Theorem 4.22] for exposition.

Theorem 3.1 (Mean Ergodic Theorem). Let $U$ be an action of $G$ on a Hilbert space $\mathcal{H}$ by unitary operators and let $x \in \mathcal{H}$. If $\left(F_{n}\right)_{n \in \mathbb{N}}$ is a Følner sequence, then

$$
\lim _{n \rightarrow \infty} \frac{1}{\left|F_{n}\right|} \sum_{g \in F_{n}} U_{g} x=P_{I} x,
$$

in the norm topology of $\mathcal{H}$, where $P_{I} x$ is the orthogonal projection of $x$ onto the closed subspace of $\mathcal{H}$ consisting of $U$-invariant vectors. 
Specializing to the unitary action associated to a $G$-system yields the following.

Corollary 3.2. Let $(X, \mu, T)$ be a $G$-system and $f \in L^{2}(\mu)$. Then

$$
\lim _{n \rightarrow \infty} \frac{1}{\left|F_{n}\right|} \sum_{g \in F_{n}} T_{g} f=P_{\mathcal{I}} f,
$$

where $P_{\mathcal{I}}$ is the orthogonal projection onto the closed subspace of $L^{2}(\mu)$ consisting of $T$ invariant functions.

We say that $(X, \mu, T)$ has finitely many ergodic components if the closed subspace of $L^{2}(\mu)$ consisting of $T$-invariant functions is finite dimensional.

Observation 3.3. If $(X, \mu, T)$ has finitely many ergodic components, then $X$ can be partitioned into $\mu$-measurable $G$-invariant sets $X_{1}, \ldots, X_{k}$ such that for each $f \in L^{2}(\mu)$, the projection $P_{\mathcal{I}} f$ can be written as

$$
\sum_{i=1}^{k}\left(\frac{1}{\mu\left(X_{i}\right)} \int f 1_{X_{i}} d \mu\right) 1_{X_{i}} .
$$

\subsection{Eigenfunctions}

We say that $f \in L^{2}(\mu)$ is an eigenfunction of $(X, \mu, T)$ (or "T-eigenfunction") if there is a character $\chi \in \widehat{G}$ such that $T_{g} f \equiv_{\mu} \chi(g) f$ for every $g \in G$. Let $\mathcal{A P}$ denote the closure of the span of the eigenfunctions of $(X, \mu, T)$ in $L^{2}(\mu)$, and write $\mathcal{W} \mathcal{M}$ for its orthogonal complement. Note that $\mathcal{W} \mathcal{M}$ is a closed, $T$-invariant subspace of $L^{2}(\mu)$.

Lemma 3.4. Let $(X, \mu, T)$ be an ergodic $G$-system and $H$ a finite index subgroup of $G$. Write $S$ for the action of $H$ obtained by restricting $T_{g}$ to $g \in H$. Then

(i) $(X, \mu, S)$ has finitely many ergodic components;

(ii) If $f \in L^{2}(\mu)$ is an S-eigenfunction, then $f$ is a finite linear combination of $T$ eigenfunctions. Thus $f \in \mathcal{A P}$.

Proof. (i) Let $B \subseteq X$ be $S$-invariant, and let $g_{1}, \ldots, g_{k}$ be coset representatives of $H$. Now

$$
X \sim_{\mu} \bigcup_{g \in G} T_{g} B \sim_{\mu} \bigcup_{i=1}^{k} T_{g_{i}} B,
$$

so $\mu(B) \geqslant \frac{1}{k}$. Thus $\inf \{\mu(B): B \subseteq X$ is $S$-invariant $\}>0$, and the algebra $\mathcal{I}_{S}$ of $\mu$ measurable $S$-invariant sets is generated (up to $\mu$-measure 0 ) by finitely many atoms $B_{1}, \ldots, B_{r}$. Now every $S$-invariant $f \in L^{2}(\mu)$ is $\mathcal{I}_{S}$-measurable, so the $1_{B_{i}}$ span the space of $S$-invariant elements of $L^{2}(\mu)$, which is therefore finite dimensional.

(ii) Let $f$ be an $S$-eigenfunction and let $g_{1}, \ldots, g_{k}$ be coset representatives of $H$ as above. Then $V$, the span of $\left\{T_{g} f: g \in G\right\}$, can be written as the span of $\left\{T_{g_{1}} f, \ldots, T_{g_{k}} f\right\}$. Thus $V$ is a finite dimensional subspace of $L^{2}(\mu)$, and $T$ induces an action of the abelian group $G$ on $V$ by unitary operators. Thus $V$ possesses a basis of $T$-eigenfunctions, and $f$ is a linear combination of these basis elements. 
Given a function $\psi: G \rightarrow \mathbb{C}$, we say that $\psi$ tends to 0 in density if for every Følner sequence $\mathbf{F}$, we have $\lim _{n \rightarrow \infty} \frac{1}{\left|F_{n}\right|} \sum_{g \in F_{n}}|\psi(g)|=0$. Equivalently, $\psi$ tends to 0 in density if for all $\varepsilon>0$, the set $A_{\varepsilon}:=\{g \in G:|\psi(g)|>\varepsilon\}$ has $d^{*}\left(A_{\varepsilon}\right)=0$.

Lemma 3.5. Let $(X, \mu, T)$ be a G-system, $f \in \mathcal{W M}$ and $h \in L^{2}(\mu)$.

(i) The correlation sequence $\psi(g):=\int f \cdot T_{g} h d \mu$ tends to 0 in density.

(ii) If $T$ has finitely many ergodic components, then $\psi_{\mathcal{I}}(g):=\left\|P_{\mathcal{I}}\left(f \cdot T_{g} h\right)\right\|_{L^{2}(\mu)}$ tends to 0 in density.

Proof. Part (i) here follows from Corollary 1.5 of [7] (cf. Proposition 2.20 and Propsition D.17 of [23]). Part (ii) follows from Part (i) and Observation 3.3.

\subsection{Reducing averages to $\mathcal{A P}$}

The main step of the proof of Theorem 2.3 is to show that the value of $I(t)$ defined in (2.3) is unaffected when each $f_{i}$ is replaced by its projection onto $\mathcal{A} \mathcal{P}$. We first prove this for $i=d$ and $i=d-1$, using Lemma 3.8 below. We then apply Corollary 3.7 to handle the remaining $f_{i}$. The asymmetry in the proof is due to the asymmetry of the hypotheses: condition (2) in Theorem 1.3 only applies to $c_{d}$ and $c_{d-1}$, and this prevents us from applying Lemma 3.8 to handle the $f_{i}$ for $i<d-1$.

We state Lemma 3.6 and Corollary 3.7 first, as their hypotheses are weaker than those of Lemma 3.8. We fix an ergodic $G$-system $(X, \mu, T)$ for the remainder of this subsection.

Lemma 3.6. Let $b \in \mathbb{Z}$. If $\chi \in \widehat{G}, f \in \mathcal{W M}$ and $b G$ has finite index in $G$, then for all $a \in \mathbb{Z}$

$$
\lim _{n \rightarrow \infty} \frac{1}{\left|F_{n}\right|} \sum_{g \in F_{n}} \chi(a g) T_{b g} f=0 \quad \text { in } L^{2}(\mu) .
$$

Proof. Let $a \in \mathbb{Z}$. Note that $g \mapsto \chi(a g)$ is a character of $G$, which we denote by $\chi^{a}$. Applying Theorem 3.1 to the unitary action $U$ on $L^{2}(\mu)$ defined by $U_{g} f=\chi(a g) T_{b g} f$, we get that the limit in (3.2) is the orthogonal projection of $f$ onto the space of $U$-invariant functions. The $U$-invariant functions are eigenfunctions for the action of $T$ restricted to $b G$, and Part (ii) of Lemma 3.4 says that these eigenfunctions are contained in $\mathcal{A P}$. Since $f \perp \mathcal{A P}$, the limit in (3.2) is 0 .

In the following corollary we abbreviate the expression $\sum_{j=1}^{d} a_{j} g_{j}$ as $\vec{a} \cdot \vec{g}$.

Corollary 3.7. Let $c_{i} \in \mathbb{Z}, i=1, \ldots, d$ so that each $c_{i} G$ has finite index in $G$. Let $h_{1}, h_{2} \in \mathcal{A P}$ and let $a_{j}, b_{j} \in \mathbb{Z}, j=1, \ldots, d$ be arbitrary. Then for all $f_{j} \in L^{\infty}(\mu)$, the value of the iterated limit

$$
Q:=\lim _{n_{1} \rightarrow \infty} \ldots \lim _{n_{d} \rightarrow \infty} \frac{1}{\left|F_{n_{1}}\right| \cdots\left|F_{n_{d}}\right|} \sum_{\substack{g_{1} \in F_{n_{1}} \\ g_{d} \in F_{n_{d}}}} T_{\vec{a} \cdot \vec{g}} h_{1} \cdot T_{\vec{b} \cdot \vec{g}} h_{2} \cdot \prod_{j=1}^{d} T_{c_{j} g_{j}} f_{j}
$$


(in the $L^{2}(\mu)$ norm) is unaffected when each $f_{j}$ is replaced by $P_{\mathcal{A P}} f_{j}$, the orthogonal projection of $f_{j}$ onto $\mathcal{A P}$.

The existence of the limit $Q$ follows from repeated application of Theorem 3.1.

Proof. We prove that the value of $Q$ remains unaffected when $f_{d}$ is replaced by $\tilde{f}_{d}$. Since $f_{d}-\tilde{f}_{d} \in \mathcal{W} \mathcal{M}$, it suffices to prove that $Q=0$ under the assumption $f_{d} \in \mathcal{W} \mathcal{M}$. A nearly identical argument will show that $f_{d-1}$ may be replaced by $\tilde{f}_{d-1}$, and so on.

Since $h_{i} \in \mathcal{A P}$ for $i=1,2$, it suffices to prove the statement when each $h_{i}$ is an eigenfunction for $(X, \mu, T)$. Thus we may assume $T_{g} h_{i}=\chi_{i}(g) h_{i}$, where $\chi_{i} \in \widehat{G}$. The innermost limit in the definition of $Q$ can be written as

$$
\lim _{n_{d} \rightarrow \infty} \beta h_{1} h_{2} \prod_{j=2}^{d} f_{j} \frac{1}{\left|F_{n_{d}}\right|} \sum_{g_{d} \in F_{n_{d}}} \chi_{1}\left(a_{d} g_{d}\right) \chi_{2}\left(b_{d} g_{d}\right) T_{c_{d} g_{d}} f_{d},
$$

where $\beta=\chi_{1}\left(\sum_{j=1}^{d-1} a_{j} g_{j}\right) \chi_{2}\left(\sum_{j=1}^{d-1} b_{j} g_{j}\right)$. The map $g_{d} \mapsto \chi_{1}\left(a_{d} g_{d}\right) \chi_{2}\left(b_{d} g_{d}\right)$ is a character of $G$, so Lemma 3.6 implies $Q=0$ when $f_{d} \in \mathcal{W M}$.

As usual for ergodic theoretic proofs of Roth's theorem, we need a van der Corput lemma. The version we use is [6, Theorem 2.12], specialized to countable discrete abelian groups; cf. [4, Lemma 4.2].

Lemma 3.8 (van der Corput lemma). Let $\left(x_{g}\right)_{g \in G}$ be a bounded collection of elements of a Hilbert space $\mathcal{H}$ and $\mathbf{F}$ be a Følner sequence. If

$$
\lim _{k \rightarrow \infty} \frac{1}{\left|F_{k}\right|} \sum_{h \in F_{k}} \lim _{n \rightarrow \infty} \frac{1}{\left|F_{n}\right|} \sum_{g \in F_{n}}\left\langle x_{g+h}, x_{g}\right\rangle=0
$$

then $\lim _{n \rightarrow \infty}\left\|\frac{1}{\left|F_{n}\right|} \sum_{g \in F_{n}} x_{g}\right\|_{\mathcal{H}}=0$.

Lemma 3.9. If $p \in L^{2}(\mu)$ and $q \in \mathcal{W M}, a, b \in \mathbb{Z}$, and both $b G$ and $(b-a) G$ have finite index in $G$, then

$$
\lim _{n \rightarrow \infty} \frac{1}{\left|F_{n}\right|} \sum_{g \in F_{n}} T_{a g} p T_{b g} q=0 \quad \text { in } L^{2}(\mu) .
$$

Proof. We will apply Lemma 3.8 with $x_{g}=T_{a g} p T_{b g} q$. First we write $\left\langle x_{g+h}, x_{g}\right\rangle_{L^{2}(\mu)}$ as

$$
\int T_{a g+a h} p \cdot T_{b g+b h} q \cdot \overline{T_{a g} p \cdot T_{b g} q} d \mu=\int \bar{p} T_{a h} p \cdot T_{(b-a) g}\left(\bar{q} T_{b h} q\right) d \mu,
$$

where we applied $T_{-a g}$ to the integrand to get the right hand side. Averaging over $g \in F_{n}$ and taking the limit, we get

$$
\begin{aligned}
\lim _{n \rightarrow \infty} \frac{1}{\left|F_{n}\right|} \sum_{g \in F_{n}}\left\langle x_{g+h}, x_{g}\right\rangle & =\int \bar{p} T_{a h} p \cdot \lim _{n \rightarrow \infty} \frac{1}{\left|F_{n}\right|} \sum_{g \in F_{n}} T_{(b-a) g}\left(\bar{q} T_{b h} q\right) d \mu \\
& =\int \bar{p} T_{a h} p \cdot P_{\mathcal{I}_{b-a}}\left(\bar{q} T_{b h} q\right) d \mu \\
& \leqslant\left\|\bar{p} T_{a h} p\right\|\left\|P_{\mathcal{I}_{b-a}}\left(\bar{q} T_{b h} q\right)\right\|,
\end{aligned}
$$


where $P_{\mathcal{I}_{b-a}}$ is the orthogonal projection onto the space of functions invariant under the action $g \mapsto T_{(b-a) g}$. Since $T$ is ergodic and $(b-a) G$ has finite index in $G$, Lemma 3.4 implies this action has finitely many ergodic components. By Lemma 3.5 we have that $\psi(h):=\left\|P_{\mathcal{I}_{b-a}}\left(\bar{q} T_{b h} q\right)\right\|$ tends to 0 in density, so

$$
\lim _{k \rightarrow \infty} \frac{1}{\left|F_{k}\right|} \sum_{h \in F_{k}} \lim _{n \rightarrow \infty} \frac{1}{\left|F_{n}\right|} \sum_{g \in F_{n}}\left\langle x_{g+h}, x_{g}\right\rangle=0,
$$

and Lemma 3.8 implies equation (3.3) holds.

\subsection{Discrete spectrum}

An ergodic $G$-system $(X, \mu, T)$ has discrete spectrum if its eigenfunctions span a dense subspace of $L^{2}(\mu)$. The Halmos-von Neumann theorem ${ }^{2}$ states that every such system is isomorphic to an ergodic group rotation system $(K, m, R)$, meaning $K$ is a compact abelian group with Haar probability measure $m$ and $R$ is given by a homomorphism $\rho: G \rightarrow K$ with dense image, so that $R_{g}(k)=k+\rho(g)$.

Multiple ergodic averages for ergodic group rotation systems can be computed as follows: if $f_{i} \in L^{\infty}(m),\left(F_{n}\right)_{n \in \mathbb{N}}$ is a Følner sequence and $c_{i} \in \mathbb{Z}$, then

$$
\lim _{n \rightarrow \infty} \frac{1}{\left|F_{n}\right|} \sum_{g \in F_{n}} \prod_{i=1}^{d} f_{i}\left(k+c_{i} \rho(g)\right)=\int_{K} \prod_{i=1}^{d} f_{i}\left(k+c_{i} s\right) d s
$$

in $L^{2}(m)$; cf. Section 3 of [16].

\subsection{Factors}

The only factor we need in this article is the Kronecker factor of an ergodic $G$-system, but we mention the general theory to fix notation. See [12], [15], or [23] for a general discussion of factors of dynamical systems.

A factor of a $G$-system $(X, \mu, T)$ is a $G$-system $(Y, \nu, S)$ together with a measurable map $\pi: X \rightarrow Y$ satisfying

$$
\mu\left(\pi^{-1} B\right)=\nu(B) \quad \text { for all } \nu \text {-measurable } B
$$

and $\pi\left(T_{g} x\right)=S_{g}(\pi(x))$ for $\mu$-almost every $x$. Note that (3.5) is equivalent to

$$
\int f d \nu=\int f \circ \pi d \mu \quad \text { for all } f \in L^{2}(\nu) .
$$

To define the Kronecker factor, we need a standard result such as [23, Theorem 1.7], which we paraphrase as follows.

Theorem 3.10. Let $(X, \mu, T)$ be an ergodic $G$-system. To every $T$-invariant $\sigma$-algebra $\mathcal{A}$ of $\mu$-measurable sets, there is a factor $(Y, \nu, S)$ with $\pi: X \rightarrow Y$ such that $A \in \mathcal{A}$ if and only if there is a $\nu$-measurable $B \subseteq Y$ with $\mu\left(A \triangle \pi^{-1} B\right)=0$. In other words, the elements of $\mathcal{A}$ are, up to $\mu$-measure 0 , the $\mu$-measurable sets of the form $\pi^{-1} B$ for $\nu$-measurable $B$.

\footnotetext{
${ }^{2}$ See $[21]$ for the case where $G=\mathbb{Z},[25]$ for general groups.
} 


\subsection{The Kronecker factor}

Every ergodic $G$-system $(X, \mu, T)$ has a factor $(K, m, R)$ with factor map $\pi: X \rightarrow K$ satisfying the following two properties:

(i) $(K, m, R)$ is an ergodic group rotation $G$-system.

(ii) Every bounded $f \in \mathcal{A} P$ is equal $\mu$-almost everywhere to a function of the form $\tilde{f} \circ \pi$, where $\tilde{f} \in L^{\infty}(m)$.

With the identification in (ii), we have

$$
f\left(T_{g} x\right)=\tilde{f}(\pi(x)+\rho(g)) \quad \text { for } \mu \text {-a.e. } x \text {, for all } g \in G \text {. }
$$

This factor can be obtained as the factor associated by Theorem 3.10 to the smallest $\sigma$ algebra of $\mu$-measurable sets with respect to which every $T$-eigenfunction is measurable.

See [16], [15], or [12] for discussion of the Kronecker factor. In [9] the Kronecker factor is discussed as the "Kronecker-Mackey factor."

We will need two standard properties of the projection map $P_{\mathcal{A P}}: L^{2}(\mu) \rightarrow \mathcal{A P}$.

- (Positivity) if $f \geqslant 0 \mu$-almost everywhere then $P_{\mathcal{A P}} f \geqslant 0 \mu$-almost everywhere, and if $f: X \rightarrow[0,1]$, then $P_{\mathcal{A P}} f(x) \in[0,1]$ for $\mu$-almost every $x$.

- (Integrals are preserved) $\int P_{\mathcal{A P}} f d \mu=\int f d \mu$ for all $f \in L^{2}(\mu)$.

These both follow from the fact that $\mathcal{A P}$ is a norm-closed algebra of functions in $L^{2}(\mu)$ containing the constant functions.

\subsection{Proof of Theorem 2.3}

We recall the statement of Theorem 2.3: let $(X, \mu, T)$ be an ergodic measure preserving $G$-system and let $\left(c_{1}, \ldots, c_{d}\right) \in \mathbb{Z}^{d}$ be coefficients satisfying hypotheses (1)-(3) of Theorem 1.3. If $f_{1}, \ldots, f_{d}: X \rightarrow[0,1]$ are measurable functions, $L: G^{d} \rightarrow[0,1]$ is defined as

$$
L\left(g_{1}, \ldots, g_{d-1} ; t\right):=\int\left(T_{t-\sum_{j<d} c_{j} g_{j}} f_{d}\right) \prod_{j<d} T_{c_{d} g_{j}} f_{j} d \mu
$$

and $I(t)$ is defined as the iterated limit

$$
I(t):=\lim _{n_{1} \rightarrow \infty} \ldots \lim _{n_{d-1} \rightarrow \infty} \frac{1}{\left|F_{n_{1}}\right| \cdots\left|F_{n_{d-1}}\right|} \sum_{g_{j} \in F_{n_{j}}} L\left(g_{1}, \ldots, g_{d-1} ; t\right)
$$

then there are

- a compact metrizable abelian group $K$ with Haar measure $m$,

- a homomorphism $\rho: G \rightarrow K$ having $\overline{\rho(G)}=K$, 
- functions $\tilde{f}_{i}: K \rightarrow[0,1]$ with $\int \tilde{f}_{i} d m=\int f_{i} d \mu$ for $i \leqslant d$

such that

$$
I(t)=\int \tilde{f}_{d}\left(k+\rho(t)-\sum_{j<d} c_{j} s_{j}\right) \prod_{j<d} \tilde{f}_{j}\left(k+c_{d} s_{j}\right) d k d s_{1} \ldots d s_{d-1} .
$$

Furthermore, if $f_{1}=\cdots=f_{d}$ then $\tilde{f}_{1}=\cdots=\tilde{f}_{d}$.

Proof. Let $\left(K, m, R_{\rho}\right)$ be the Kronecker factor of $(X, \mu, T)$ with factor map $\pi: X \rightarrow K$. As above, let $\mathcal{A P}$ denote the closure of the span of the eigenfunctions of $(X, \mu, T)$, so that every bounded $f \in \mathcal{A P}$ can be written as $\tilde{f} \circ \pi$, where $\tilde{f} \in L^{\infty}(m)$.

The main step of the proof is to show that the innermost limit in the definition of $I(t)$ is unaffected when $f_{d}$ is replaced by $P_{\mathcal{A P}} f_{d}$. To prove this it suffices to prove that the innermost limit is 0 when $P_{\mathcal{A P}} f_{d}=0$, i.e. when $f_{d} \in \mathcal{W} \mathcal{M}$. Assuming $f_{d} \in \mathcal{W} \mathcal{M}$, we write the innermost average in (3.9) as

$$
\frac{1}{\left|F_{n}\right|} \sum_{g_{d-1} \in F_{n}} \int T_{t-\sum_{j<d} c_{j} g_{j}} f_{d} \cdot T_{c_{d} g_{d-1}} f_{d-1} \cdot \prod_{j<d-1} T_{c_{d} g_{j}} f_{j} d \mu .
$$

We rewrite the first factor $T_{t-\sum_{j<d} c_{j} g_{j}} f_{d}$ as $T_{-c_{d-1} g_{d-1}} f_{d}^{\prime}$, where $f_{d}^{\prime}:=T_{t-\sum_{j<d-1} c_{j} g_{j}} f_{d}$. Now $f_{d}^{\prime} \in \mathcal{W} \mathcal{M}$, since $f_{d} \in \mathcal{W} \mathcal{M}$ and $\mathcal{W} \mathcal{M}$ is a $T$-invariant subspace of $L^{2}(\mu)$. The above expression can now be written as

$$
\int\left(\frac{1}{\left|F_{n}\right|} \sum_{g_{d-1} \in F_{n}} T_{-c_{d-1} g_{d-1}} f_{d}^{\prime} \cdot T_{c_{d} g_{d-1}} f_{d-1}\right) \prod_{j<d-1} T_{c_{d} g_{j}} f_{j} d \mu .
$$

Noting that $\left(c_{d}-\left(-c_{d-1}\right)\right) G$ has finite index in $G$ by hypothesis (2) of Theorem 1.3, we may apply Lemma 3.9 to the average in parentheses in (3.11) and conclude that it converges to 0 in $L^{2}(\mu)$ as $n \rightarrow \infty$.

The same argument shows that $f_{d-1}$ in (3.9) can be replaced by $P_{\mathcal{A P}} f_{d-1}$ without affecting the innermost limit. Thus we may assume $f_{j} \in \mathcal{A P}$ for $j=d$ and $d-1$. Under this assumption, Corollary 3.7 allows us to replace the remaining $f_{j}, 1 \leqslant j<d-1$ with $P_{\mathcal{A P}} f_{j}$ without affecting the limit in (3.9). Having replaced each $f_{j}$, we write $P_{\mathcal{A P}} f_{j}$ as $\tilde{f}_{j} \circ \pi$, and note that equation (3.7) means $T_{h} P_{\mathcal{A P}} f_{j}(x)=\tilde{f}_{j}(\pi(x)+\rho(h))$ for $\mu$-almost every $x$. We evaluate the innermost limit in (3.9) using (3.6) and (3.4) to get

$$
\begin{aligned}
& \lim _{n_{d-1} \rightarrow \infty} \frac{1}{\left|F_{n_{d-1}}\right|} \sum_{g_{d-1} \in F_{n_{d-1}}} \int \tilde{f}_{d}\left(k+\rho\left(g-\sum_{j<d} c_{j} g_{j}\right)\right) \prod_{j<d} \tilde{f}_{j}\left(k+\rho\left(c_{d} g_{j}\right)\right) d k \\
& =\int \tilde{f}\left(k+\rho\left(g-\sum_{j<d-1} c_{j} g_{j-1}\right)+c_{d-1} s_{d-1}\right) \prod_{j<d-1} \tilde{f}_{j}\left(k+\rho\left(c_{d} g_{j}\right)\right) \tilde{f}_{d-1}\left(k+c_{d} s_{d-1}\right) d k d s_{d-1} .
\end{aligned}
$$

The same evaluation for the averages over $g_{j} \in F_{n_{j}}, j=d-2, j=d-3, \ldots, j=1$, will simplify the remaining limits in (3.9) to obtain (3.10).

To justify the last sentence of Theorem 2.3, note that our definition of $\tilde{f}_{i}$ implies $\tilde{f}_{i}=\tilde{f}_{j}$ whenever $f_{i}=f_{j}$. 


\section{Problems}

Theorems 1.2 and 1.3 suggest the following problems.

Problem 4.1. Under the hypotheses of Theorem 1.3, provide quantitative bounds on the rank and radius of a basic Bohr neighborhood contained in $\vec{c} \cdot A$ in terms of $d^{*}(A)$ and the indices of the subgroups $c_{i} G$ in $G$.

Question 4.2. Can hypothesis (2) in Theorem 1.3 be omitted without affecting the conclusion?

Here is a specific instance where hypotheses (1) and (3) hold but hypothesis (2) fails.

Problem 4.3. Prove or disprove: if $A \subseteq \mathbb{F}_{3}^{\omega} \times \mathbb{F}_{5}^{\omega}$ has $d^{*}(A)>0$ and $\vec{c}=(1,2,4,-7)$, then $\vec{c} \cdot A$ is a Bohr neighborhood of 0 .

Our proof of Theorem 1.3 uses hypothesis (2) to evaluate the limit in (3.11) above. This step invokes Lemma 3.9, which is a specialization of [4, Theorem 4.3] (see also [1, Theorem 3.1] and especially [2, Proposition 3.5]). Without hypothesis (2), we cannot guarantee that the subgroup $(b-a) G$ in Lemma 3.9 has finite index in $G$. One could invoke the more general Theorem 4.3 from [4] in place of Lemma 3.9, but this would require a more intricate analysis of the limit in (3.11), due to phenomena discussed in Remark 3.2 of [2].

Remark 4.4. Our Theorem 1.3 does not recover Følner's and Bogoliouboff's theorems for groups where $2 G$ has infinite index in $G$, such as $\mathbb{Z}^{\omega}$ and $\mathbb{F}_{2}^{\omega}$, since $\vec{c}=(1,-1,1,-1)$ does not satisfy hypothesis (2) in this case. However, in these two groups, the only nontrivial instances of coefficients $c_{1}, \ldots, c_{d}$ where each $c_{i} G$ has finite index in $G$ and the exponent of $G$ divides $\sum c_{i}$ are those where $d$ is even and every $c_{i}$ is 1 or -1 . That $\vec{c} \cdot A$ is a Bohr neighborhood of 0 in this case is already a consequence of Følner's theorem, as such $\vec{c} \cdot A$ will include a subset of the form $\vec{b} \cdot A+\vec{d} \cdot A$, where $\vec{b}=(1,-1,1,-1)$ and the entries of $\vec{d}$ sum to 0 . The situation may be more complicated in other groups where $2 G$ has infinite index in $G$, and we hope that a positive resolution of Question 4.2 will result in a proof that recovers Theorem 1.3 and all instances of Følner's theorem for abelian groups simultaneously.

Our Theorem 1.3 does recover the special cases of Følner's and Bogoliouboff's theorems where $2 G$ has finite index in $G$, such as finitely generated abelian groups and vector spaces over fields of odd characteristic or characteristic 0 .

\section{Acknowledgement}

An anonymous referee for The Electronic Journal of Combinatorics provided several corrections and improvements to exposition. 


\section{References}

[1] Ethan Ackelsberg, Vitaly Bergelson, and Andrew Best. Multiple recurrence and large intersections for abelian group actions. Discrete Anal., 2021:18, 91pp, 2021.

[2] Ethan Ackelsberg, Vitaly Bergelson, and Or Shalom. Khintchine-type recurrence for 3-point configurations. arXiv:2201.03924, January 2022.

[3] Mathias Beiglböck, Vitaly Bergelson, and Alexander Fish. Sumset phenomenon in countable amenable groups. Adv. Math., 223(2):416-432, 2010.

[4] Vitaly Bergelson, Randall McCutcheon, and Qing Zhang. A Roth theorem for amenable groups. Amer. J. Math., 119(6):1173-1211, 1997.

[5] Vitaly Bergelson and Andreu Ferré Moragues. An ergodic correspondence principle, invariant means and applications. Isr. J. Math., 2021. published online at https://doi.org/10.1007/s11856-021-2233-y

[6] Vitaly Bergelson and Joel Moreira. Van der Corput's difference theorem: some modern developments. Indag. Math. (N.S.), 27(2):437-479, 2016.

[7] Vitaly Bergelson and Joseph Rosenblatt. Mixing actions of groups. Illinois J. Math., 32(1):65-80, 1988.

[8] Vitaly Bergelson and Imre Z. Ruzsa. Sumsets in difference sets. Israel J. Math., 174:1-18, 2009.

[9] Michael Björklund and Alexander Fish. Approximate invariance for ergodic actions of amenable groups. Discrete Anal., 2019:6, 56pp, 2019.

[10] Michael Björklund and John T. Griesmer. Bohr sets in triple products of large sets in amenable groups. J. Fourier Anal. Appl., 25(3):923-936, 2019.

[11] N. Bogolioùboff. Sur quelques propriétés arithmétiques des presque-périodes. Ann. Chaire Phys. Math. Kiev, 4:185-205, 1939.

[12] Manfred Einsiedler and Thomas Ward. Ergodic theory with a view towards number theory, volume 259 of Graduate Texts in Mathematics. Springer-Verlag London, Ltd., London, 2011.

[13] Erling Følner. Note on a generalization of a theorem of Bogolioùboff. Math. Scand., 2:224-226, 1954.

[14] Alan H. Forrest. Recurrence in dynamical systems: A combinatorial approach. ProQuest LLC, Ann Arbor, MI, 1990. Thesis (Ph.D.)-The Ohio State University.

[15] H. Furstenberg. Recurrence in ergodic theory and combinatorial number theory. Princeton University Press, Princeton, N.J., 1981. M. B. Porter Lectures.

[16] Harry Furstenberg. Ergodic behavior of diagonal measures and a theorem of Szemerédi on arithmetic progressions. J. Analyse Math., 31:204-256, 1977.

[17] Alfred Geroldinger and Imre Z. Ruzsa. Combinatorial number theory and additive group theory. Advanced Courses in Mathematics. CRM Barcelona. Birkhäuser Verlag, Basel, 2009. Courses and seminars from the DocCourse in Combinatorics and Geometry held in Barcelona, 2008. 
[18] John T. Griesmer. Bohr topology and difference sets for some abelian groups. arXiv:1608.01014, November 2017.

[19] John T. Griesmer. Separating Bohr denseness from measurable recurrence. Discrete Anal., 2021:9, 20pp, 2021.

[20] John T. Griesmer. Special cases and equivalent forms of Katznelson's problem on recurrence. arXiv:2108.02190, August 2021.

[21] Paul R. Halmos and John von Neumann. Operator methods in classical mechanics. II. Ann. of Math. (2), 43:332-350, 1942.

[22] Norbert Hegyvári and Imre Z. Ruzsa. Additive structure of difference sets and a theorem of Følner. Australas. J. Combin., 64:437-443, 2016.

[23] David Kerr and Hanfeng Li. Ergodic theory. Springer Monographs in Mathematics. Springer, Cham, 2016. Independence and dichotomies.

[24] Igor Kříž. Large independent sets in shift-invariant graphs: solution of Bergelson's problem. Graphs Combin., 3(2):145-158, 1987.

[25] George W. Mackey. Ergodic transformation groups with a pure point spectrum. Illinois J. Math., 8:593-600, 1964.

[26] Randall McCutcheon. Elemental methods in ergodic Ramsey theory, volume 1722 of Lecture Notes in Mathematics. Springer-Verlag, Berlin, 1999. 